\title{
ESTIMATION OF POPULATION SIZE AND PROBABILITIES OF SURVIVAL AND DETECTION IN MEAD'S MILKWEED
}

\author{
Norman A. Slade, $, 2,2,4$ Helen M. Alexander, ${ }^{2}$ And W. Dean Kettle ${ }^{3}$ \\ ${ }^{1}$ Natural History Museum, 1345 Jayhawk Blvd., University of Kansas, Lawrence, Kansas 66045-7561 USA \\ ${ }^{2}$ Department of Ecology \& Evolutionary Biology, University of Kansas, Lawrence, Kansas 66045-7534 USA \\ ${ }^{3}$ Kansas Biological Survey, University of Kansas, Lawrence, Kansas 66047-2906 USA
}

\begin{abstract}
Statistical similarities exist between estimating numbers of cryptic animals and of inconspicuous plants. Without flowers, Mead's milkweed (Asclepias meadii) is an unobtrusive prairie plant, and most plants flower irregularly. We used maximum likelihood to estimate probabilities of survival and detection for milkweeds in annual censuses during 1992-1999. Initially, we assumed no recruitment and fit models to all data and to flowering plants only. Because plants were marked when located, probabilities of resighting exceeded those of initial discovery. Plants were most likely to flower and be detected in years when the prairie was burned. We marked 177 plants in eight years but estimated the 1992 population to be 337 or 191 plants with 166 or 121 surviving to 1999, depending on the data set. Thus, estimated population size exceeded number of plants seen. Estimated annual survival probability was generally $\geq 0.95$, but aggregate survival over eight years predicted rapid extinction without recruitment. When we included recruitment, estimates of survival changed little, and estimated population size varied between 118 and 147 individuals. Discovery of new plants in two additional years (2000 and 2001) appeared to be consistent with required recruitment, but simple counts of these plants did not track population trends.

Key words: Asclepias meadii; capture-recapture; census; estimation of population size; maximum likelihood; Mead's milkweed; probability of detection; survival.
\end{abstract}

\section{INTRODUCTION}

Counting or estimating the numbers of individuals is one of the central tasks of population ecologists. For many groups of animals, complete counts are impossible or impractical, and total numbers must be inferred from the number of individuals that are actually seen or captured over a series of censuses. If the proportion of surviving individuals that have been captured, marked, and subsequently resighted or recaptured is low (i.e., low detection probability), the estimated population size will be much larger than the count at any single census. A number of methods have been developed to estimate population size based on various statistical models and assumptions regarding population processes (Pollock et al. 1990). We can treat populations as closed, with no births or deaths occurring; alternatively, they can be open to recruitment and loss. Individuals may all be equally likely to be encountered, or probabilities of detection may vary with time or among individuals.

Plants have been assumed easier to count than animals because plants are stationary. However, many plants are difficult to detect for a variety of reasons. For instance, the small yellow lady's slipper orchid (Cypripedium calceolus spp. parviflorum) may undergo periods of dormancy when it has no aboveground struc-

Manuscript received 2 January 2002; revised 29 July 2002; accepted 2 August 2002. Corresponding Editor: E. S. Menges.

${ }^{4}$ E-mail: slade@ku.edu tures (Shefferson et al. 2001); other examples are discussed in Lesica and Steele (1994). Similarly, the focus of this study, Mead's milkweed (Asclepias meadii Torr. ex A. Gray), may be undetected in some years either because it does not produce aboveground structures or it can exist only as small, nonflowering stems embedded in dense tallgrass prairies (Alexander et al. 1997). Recently, ecologists have used capture-recapture analyses, developed for animals, to estimate population size and survival rates for such cryptic plants (Alexander et al. 1997, Shefferson et al. 2001).

In our previous work (Alexander et al. 1997), we used mark-recapture methodology to estimate population size for a population of Mead's milkweed in a 4.5-ha area using data from 1992 through 1995. With our four-year data set, an assumption of a closed population seemed reasonable, because plants had quite high rates of survival ( $96.3 \%$ per annum) and low rates of reproduction. That paper reported that the estimated population size was $\geq 50 \%$ greater than the total number of plants that had been marked in four annual censuses. We speculated that, if recruitment were truly negligible, continued censuses should reveal more plants but that the total number of marked plants would reach an asymptote through time. Our study has continued, making the assumption of no mortality more tenuous. In this paper, we include data from an additional four years (total span of eight years, 1992-1999) and estimate initial population size (e.g., in the year 1992) and subsequent rates of decrease using a model 
of variable rates of survival and detection that depend on management treatment. That is, we allow the population to be open and plants to be lost, but initially we retain the assumption of no recruitment to the population, based on low seed production (Kettle et al. 2000) and our inability to observe seedlings. We use this model to compare estimates of population size and survival with those from the first four years of study (Alexander et al. 1997), and we examine the numbers of newly detected plants for indications that we are reaching an asymptote in total numbers of plants that have been discovered. However, the assumption of no recruitment overestimates survival if there actually is some recruitment. Therefore, we also estimate survival based solely on the frequency of resightings, which is independent of estimates of recruitment or population size. We then estimate the level of recruitment needed to sustain the population and compare predictions with the numbers of newly discovered plants in 2000 and 2001. Finally, we used a model of recruitment and mortality to estimate survival and population size simultaneously.

\section{MethodS \\ Study organism and site}

Mead's milkweed is a clonal perennial of tallgrass prairies in the central United States. These milkweeds are long-lived; for example, plants have persisted for decades in mowed hay meadows, where sexual reproduction is prevented by the mowing schedule (Bowles et al. 1998). Betz (1989) suggested that individual plants might live for a century or more. Further, there are low rates of seed production on our 4.5-ha study area (Kettle et al. 2000). Over an eight-year span, $<20 \%$ of flowering plants produced seeds; and, for five of these years, fewer than five follicles (seed pods) were produced on the entire site. Seedlings may take $15 \mathrm{yr}$ to reach reproductive size (Bowles et al. 2001). Burning seems to increase production of both flowers and follicles. Mead's milkweed was once found throughout Midwestern prairies, but is now federally listed, due in part to destruction of habitat (Betz 1989, Bowles et al. 1998). In this paper, we follow Alexander et al. (1997) and Kettle et al. (2000) in referring to clusters of stems within $1.25 \mathrm{~m}$ as a single plant. At our site, extensive clonal spread does not occur. The maximum span of a plant was less than $1.7 \mathrm{~m}$, and plants were usually separated by several meters. Our choice of 1.25 $\mathrm{m}$ was arbitrary, but based on knowledge that rhizomes can extend up to $1 \mathrm{~m}$ (M. L. Bowles, personal communication).

Our study site is on the University of Kansas Field Station and Ecological Reserves, $12 \mathrm{~km}$ northeast of Lawrence, Kansas, USA (Alexander et al. 1997, Kettle et al. 2000). Most plants were located on the Rockefeller Native Prairie tract, which has never been plowed, but a few plants occur on an adjoining area of reseeded prairie. The prairie has been mowed and burned periodically since at least 1930 . Most recently, it has been burned in March or April of even numbered years, before the emergence of Mead's milkweeds, to discourage the growth and spread of woody plants (Kettle et al. 2000).

\section{Census}

Annual surveys for individual plants were conducted from 1992 to the present (see Alexander et al. 1997 and Kettle et al. 2000 for details). In early June, field crews systematically searched the entire area. Plants were marked with flags when found, and locations were plotted using a preexisting coordinate system. Subsequent surveys involved intensive search at each location where a plant had been found, as well as systematic search for new plants. Annual surveys were conducted using consistent methods and sampling intensity; one of us (W. D. Kettle) oversaw all surveys, whereas G. Pittman led the field crews since 1994 and made decisions regarding identity of individual plants. Despite intensive search, plants were not seen in every year between their first and last discovery. Hence each individual plant generated a "capture history"; that is, each plant was represented by a series of ones and zeros denoting whether it had been seen or not in a particular year (see the Appendix).

Annual survey data were explored in several ways. First, we examined a plot of cumulative number of plants discovered over time for evidence of an asymptote. Second, we plotted the total number of plants seen in each year, looking for evidence of population persistence or decline. Third, in our earlier paper (Alexander et al. 1997), we were intrigued by whether the very large number of newly discovered plants in 1994 (an unusual year in which burning was preceded by extraordinarily heavy rainfall in 1993; Kettle et al. 2000) constituted a subclass of the population that flowered infrequently. Thus, we compared the subsequent frequency of flowering of plants first seen in 1994 with those of plants seen in 1994 and before by using $\chi^{2}$ and Kruskal-Wallis tests.

\section{Models}

Because all living plants were not seen in each survey, we used statistical methods, originally developed for estimating survival and numbers from capture-recapture of animals (see Nichols [1992] for an overview), to estimate population size and yearly survival for Mead's milkweed. In our previous study, spanning four years, we assumed that no plants died or were recruited, and used statistical models for closed populations (Otis et al. 1978, White et al. 1982) to estimate population size (Alexander et al. 1997). With an additional four years of data, the assumption of no mortality was questionable, so we wanted to estimate survival and initial (1992) population size simultaneously, while retaining the assumption of no recruitment. We 


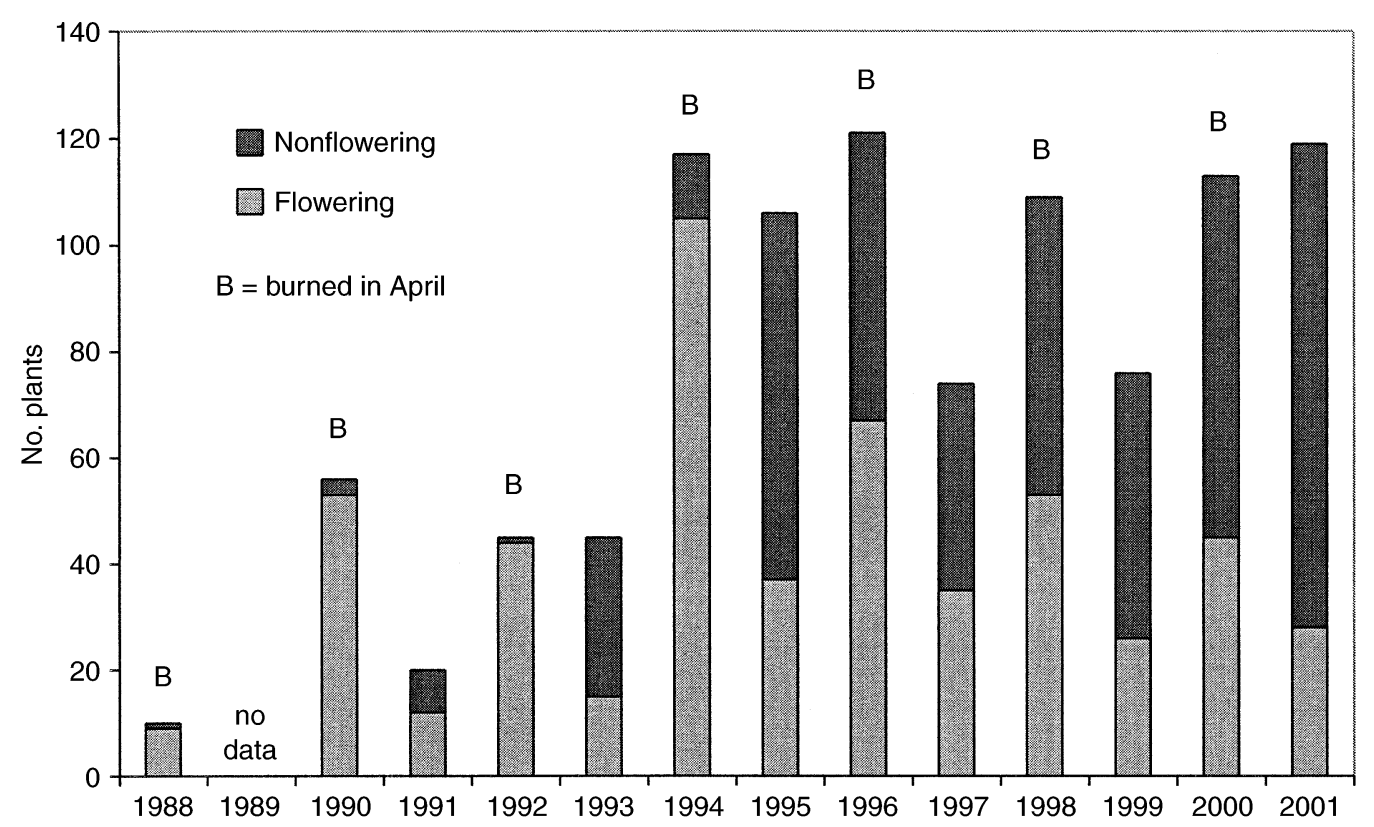

FIG. 1. The number of flowering and nonflowering plants of Asclepias meadii detected during 1988-2001. In even years (noted by B), the prairie was burned in April, prior to the emergence of stems of A. meadii. We included data from 19881991 surveys (see Kettle et al. 2000), which were less intensive but similar to those in this study.

estimated population size in 1999 by multiplying initial size by subsequent survival rates. The proportion of organisms that were alive but overlooked or dormant in a single survey was estimated from the proportion of organisms that were missed in that survey but known to survive because they were found subsequently. Thus proportional survival is a population statistic, and as in animal studies (Pollock et al. 1990), individual organisms that are not seen need not be categorized as dead, dormant, or undetected for other reasons. Because all plants that were seen were assumed to be alive at the start of the study, our procedure produced estimates of maximum survival and minimum probabilities of discovery. That is, a plant first seen in 1995 was counted as surviving without detection from 1992 to 1995.

To understand our modeling exercise, it is important to briefly summarize our earlier results and approach. In both Alexander et al. (1997) and Kettle et al. (2000), we found probabilities of discovery of plants varied temporally, and more plants were detected in years with spring burns (Fig. 1). Detection probability also varied among plants, and probabilities of resighting plants were greater than probabilities of initial discovery. Our first statistical model $\left(\mathrm{M}_{\mathrm{tbh}}\right)$ in Alexander et al. (1997) included variation among plants and years and between first and subsequent sightings, but did not allow estimation of population size. We were able to simplify that model by using counts of flowering plants (i.e., 1 's in a capture history denote a flowering plant, whereas 0's refer to both nonflowering and undetected plants), which reduced the discrepancy between prob- abilities of first and subsequent sightings and allowed estimation of population size.

To extend this approach for an additional four years, we retained some of the above biological features: i.e., that initial probabilities of finding plants were much lower than probabilities of resighting, that plants would be more likely to be found in growing seasons following spring burning, and that recruitment was negligible. We also expected that probabilities of survival probably differed from year to year, but we had no hypotheses as to the impact of burning or other environmental influences on survival. This led to a statistical model of year-specific probabilities of survival and four probabilities of "capture" - initial discovery in years with and without burns and subsequent discovery, with and without fire. Unfortunately, we were unable to fit such a model using MARK (White 1999) or other software packages that were available to us. Therefore, we wrote a BASIC program to find the maximum-likelihood estimators (MLEs) for the parameters of our statistical model (see the Appendix). Following our earlier approach (Alexander et al. 1997), we analyzed the data set with all plants, as well as a second data set in which only flowering plants were noted as present. Use of a flowering data set lessened the distinction between initial discovery and resighting, and we fit a model with only three probabilities of being seen-years with spring burning, years without fire, and 1994, the unusual year noted earlier. Both of our approaches (all plants and only flowering plants) were designed to estimate the size of the same population, i.e., the number 


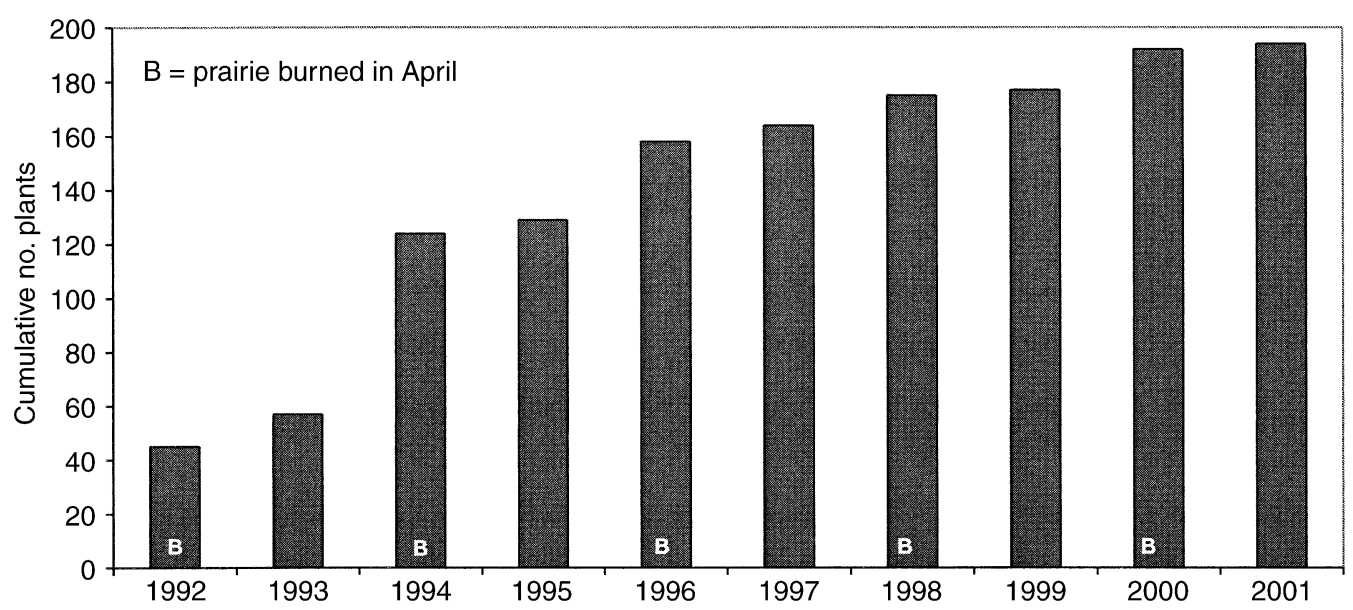

FIG. 2. The cumulative number of plants of Asclepias meadii detected during 1992-2001. In even years (noted by B), the prairie was burned in April, prior to the emergence of stems of A. meadii.

of mature plants (but not seedlings or juveniles, which cannot be detected in our surveys).

We generated jackknifed standard errors for all estimates by sequentially omitting each plant that was observed from a data set, generating MLEs, and calculating the standard deviation among estimates for each parameter. When we jackknifed the entire data set, several capture histories led to excessively large estimates of population size, and we arbitrarily set these estimates to 1177 ( $=1000$ more plants than we actually observed). We tested our computer program by modifying it to fit several models that we could also fit in MARK, and we obtained identical (to three decimal places) results using both programs. Because the order of grid search through the 11- or 12-dimensional parameter space might have influenced our result, we fit each model five additional times using random orders for the parameters other than population size. Each run gave identical MLEs.

We used the estimated survival rates and population size from the period 1992-1999 to determine the recruitment needed to sustain the population; we then compared it to the observed new plants in 2000 and 2001. Because the entry of new plants into the population meant that our previous estimates of survival might be positively biased, we also estimated probabilities of resighting and survival based solely on the frequency of resightings using procedures developed by Cormack (1964), Jolly (1965), and Seber (1965) and available in program MARK (White 1999). The Cormack-Jolly-Seber (C-J-S) estimates of survival are independent of estimates of recruitment or population size, but they do not provide estimates of initial discovery or of population size (which are interrelated). Finally, we also estimated population size using a standard Jolly-Seber model (Model A), which allows recruitment, but does not distinguish between initial and subsequent probabilities of detection. Because both the C-J-S and standard Jolly-Seber analyses require data subsequent to the year of interest, we included surveys from 2000 and 2001 to estimate 1998-1999 survival; thus those analyses are performed on slightly different data sets than the previous models.

\section{RESULTS}

The rate of increase in the cumulative number of plants observed has slowed in recent years, but new plants were found in both 2000 and 2001 (15 and 2 plants respectively; Fig. 2). Note that in burn years (even years) higher numbers of plants are seen and more new plants are discovered (Figs. 1 and 2). The total number of plants seen fluctuated widely through the study period (Fig. 1). Plants that first flowered in 1994 and those that flowered in 1994 and before did not differ in frequency of flowering during 1995-1999 (Kruskal-Wallis $H=0.39, P=0.534$ ) nor in the temporal patterns of flowering over this period (analyses of frequencies of capture histories; $\chi^{2}=17.23$, df $=$ $19, P=0.57)$.

As we anticipated, estimated probabilities of discovering plants were greater in growing seasons that followed spring burning, and probabilities of resighting were much higher than probabilities of initial discovery for data from all plants (Table 1). Estimates of survival were relatively high, but varied among years and differed depending on whether flowering or all plants were counted as being present. Our C-J-S estimates of survival, which were independent of estimates of population size, were similar to those of our other models (Table 1). Our maximum-likelihood procedure using all plants generated an estimate of 337 plants in 1992, with 166 remaining in 1999. Respective estimates from data on flowering plants only were 191 and 121, respectively. We also fit a standard Jolly-Seber model with time-specific rates of survival and detection (White 1999), which produced an estimate of mean population size over the eight-year period of 123 (1 SE $\approx 5$; 1992-1999 numbers are 57, 126, 118, 147, 141, 
TABLE 1. Estimated probabilities of survival and sighting and estimated population size using data from all plants and from flowering plants only using a model of no recruitment and the Cormack-Jolly-Seber (C-J-S) model to estimate survival with recruitment independent of population size.

\begin{tabular}{|c|c|c|c|c|c|c|}
\hline \multirow[b]{2}{*}{ Parameter } & \multicolumn{2}{|c|}{ All plants } & \multicolumn{2}{|c|}{ Flowering plants } & \multicolumn{2}{|c|}{ C-J-S all plants } \\
\hline & Estimate & $1 \mathrm{SE}$ & Estimate & $1 \mathrm{SE}$ & Estimate & $1 \mathrm{SE}$ \\
\hline 1992 numbers & 337 & 24828 & 191 & 13.98 & $\ldots$ & $\ldots$ \\
\hline 1992-1993 survival & 0.953 & 0.0663 & 0.999 & 0.2049 & 0.935 & 0.0373 \\
\hline 1993-1994 survival & 0.999 & 0.0000 & 0.999 & 0.0000 & 1.000 & 0.0000 \\
\hline 1994-1995 survival & 0.922 & 0.0736 & 0.949 & 0.2015 & 0.928 & 0.0296 \\
\hline 1995-1996 survival & 0.887 & 0.1251 & 0.869 & 0.3737 & 0.891 & 0.0352 \\
\hline 1996-1997 survival & 0.858 & 0.1240 & 0.771 & 0.3524 & 0.863 & 0.0361 \\
\hline 1997-1998 survival & 0.868 & 0.0761 & 0.999 & 0.0000 & 0.878 & 0.0388 \\
\hline 1998-1999 survival & 0.847 & 0.0870 & 0.999 & 0.0010 & 0.848 & 0.0686 \\
\hline Discovery with spring burn & 0.179 & 0.4635 & 0.352 & 0.0890 & $\ldots$ & $\ldots$ \\
\hline Discovery without fire & 0.039 & 0.0240 & 0.186 & 0.0787 & $\ldots$ & $\ldots$ \\
\hline Resighting with spring burn & 0.910 & 0.1000 & $\ldots$ & $\ldots$ & 0.908 & 0.0200 \\
\hline Resighting without fire & 0.753 & 0.0412 & $\ldots$ & $\ldots$ & 0.752 & 0.0279 \\
\hline Discovery in 1994 & $\ldots$ & $\ldots$ & 0.551 & 0.1817 & $\ldots$ & $\ldots$ \\
\hline
\end{tabular}

Notes: The first two columns of standard errors were estimated by jackknifing, i.e., omitting single plants from the data set. Ellipses indicate parameters that were not in the likelihood model.

142,129 , respectively). In any case, estimated population sizes exceeded the observed number of plants (Table 1, Fig. 1).

\section{Discussion}

For cryptic, long-lived plants, long-term monitoring of populations is essential for both estimation of population size in any one year and interpretation of population trajectories. Our data reveal that probabilities of flowering and discovery of plants vary among years. For example many more plants were found in years following burning. Consequently, simple counts of plants seen over a series of years are less than the actual numbers of plants present. Furthermore, counts based on annual surveys are only loosely related to estimated population size; hence they are weak indices of population size (Nichols 1992, Slade and Blair 2000). This dilemma emphasizes the necessity of using statistical models to estimate numbers. In this study, we used models suggested by field observations, rather than our earlier use of models chosen through a selection procedure (Alexander et al. 1997), but either approach is an improvement on using simple numbers as an estimate or index of population size.

After reporting a closed population estimate of 219 plants using a four-year data set (Alexander et al. 1997), we were motivated to continue our study for four more years to test our prediction that the cumulative number of plants seen should reach an asymptote near 220 plants. Such an asymptote seems reasonable through the year 1999 (Fig. 2). Applying the same closed population model $\left(\mathrm{M}_{\mathrm{th}}\right.$, which incorporates variation in time and among plants) used by Alexander et al. (1997) to the entire eight-year data set for flowering plants yielded an estimate of 239 with a standard error of 17.6. But our estimates of survival (Table 1) were low enough to negate the assumption of a closed population over an eight-year period. Our maximum-likelihood procedure, using counts of all plants, generated an estimate of 337 plants in 1992, with 166 surviving until 1999. Respective estimates from the flowering plant data set were 191 and 121 . Considering the respective standard errors $(>20000$ vs. $<14)$, the latter estimates are much less sensitive to the frequency of individual capture histories, and we were more comfortable with the estimates from the flowering plants. Estimates of population size using all plants were particularly sensitive to frequencies of several capture histories involving plants first seen in 1994. However, we found no evidence that plants first flowering in 1994 are unusual in subsequent flowering frequency or survival. Because our model of survival ignored recruitment, our estimates of numbers, especially early in the study, are probably positively biased-all plants seen throughout the study were assumed to be present in 1992. Subsequent data (2000 and 2001) have cast further doubt on the scenario of sustained decline. In contrast, the standard Jolly-Seber model permitted unlimited recruitment, so many plants discovered after 1992 were attributed to recruitment. This model resulted in lower estimates of population size that averaged only 27 individuals greater than the number of plants seen. In years following spring burning, counts were within four, but not two, standard errors of estimated numbers.

The geometric mean of our estimates of survival rate (between 0.90 and 0.93, depending on model, Table 1) seems at odds with higher rates implied by Betz (1989), but direct comparison is difficult. He stated that plants observed in 1965 were still present two decades later, but sample sizes are unclear. Mean annual survival of 0.90 predicts only $12 \%$ survival for $20 \mathrm{yr}$ or more, and 0.93 is consistent with $25 \%$ of plants surviving that long. Nevertheless, persistence of plants in hay meadows with no sexual reproduction for decades supports 
higher survival than we found. Tecic et al. (1998) reported that the few fire-managed populations they studied had fewer ramets per genet than hay meadows; one hypothesis is that mowing produces more stems per plant, and that this results in greater survivorship.

Even our highest estimates of survival are so low as to predict loss of $99 \%$ of plants in $60 \mathrm{yr}$ unless there is modest recruitment. Given a 0.93 survival rate, per capita annual recruitment of $\sim 7 \%$ would be needed to maintain the population. If our population is between 123 (the average from the standard Jolly-Seber model) and 144 (the average of the two 1999 estimates from models ignoring recruitment), this would require 9 to 10 recruits per year, and if the proportion of marked plants is to remain constant, we should discover 9 or 10 new plants per year. Since this calculation is based on analyses of 1992-1999 data, we examined the number of newly discovered plants in two independent years, 2000 and 2001. We found a total of 15 new plants in 2000 (a burn year) and 2 in 2001. Hence our recent rate of discovery of new plants, while not high enough to assure population stability, is potentially consistent with a recruitment rate sufficient to sustain our population. Similarly, our data could indicate a stable population (Fig. 1), given the similarity in maximum number of plants seen over several years; but those data also are consistent with a declining population in which an increasing proportion of plants are found and marked through time.

Each of our results is model dependent. Originally, when we assumed a closed population, we predicted a population size of $>200$ plants (Alexander et al. 1997). Collecting more data and recognizing significant but variable rates of mortality led to estimates of initial (1992) populations $>200$, but current numbers of $\leq 166$. Finally, using models with both recruitment and mortality led to a scenario of populations that are more stable, varying between 120 and 145 individuals. While the estimates of numbers from this latter model appear most plausible, Jolly-Seber overestimates probabilities of detection, because that model ignores the fact that probabilities of discovery of new plants are lower than those of rediscovery of plants marked by flags. Overestimating probabilities of detection leads to underestimating population size (Nichols 1992); hence the Jolly-Seber estimates of population size are likely negatively biased. True population sizes probably fall between the estimates from the different models.

At this point, we can make predictions that can be directly tested with subsequent censuses. Under the scenario of insufficient recruitment, we should find fewer and fewer new plants in future years. However, if the population is stationary, the number of new plants per year should stabilize, and we will continue to find new plants each year, or at least each burn year. Finally, it is also possible that recruitment in the population is episodic, and that years such as 1994 in which large numbers of unmarked plants were discovered will re- occur. Thus researchers studying long-lived, cryptic plants share similar problems with zoologists who routinely must estimate population abundances from partial censuses-one can not rely on simple counts, and the lack of precision in estimating numbers in a single year makes it difficult to interpret population trajectories.

\section{ACKNOWLEDGMENTS}

We particularly thank G. Pittman, who has done the majority of Mead's milkweed monitoring in recent years. Our long-term data set has required fieldwork by numerous people, including K. Kindscher, A. Reber, J. Campbell, A. McMillan, G. Ward, T. Daughterty, B. Johanning, J. Phelan, and A. Schrag. The Kansas Biological Survey provided financial support. We benefited greatly from comments of two anonymous reviewers and the editor.

\section{Literature Cited}

Alexander, H. M., N. A. Slade, and W. D. Kettle. 1997. Application of mark-recapture models to estimation of the population size of plants. Ecology 78:1230-1237.

Betz, R. F. 1989. Ecology of the Mead's milkweed (Asclepias meadii Torrey). Pages 187-191 in T. B. Bragg and J. Stubbendieck, editors. Proceedings of the Eleventh North American Prairie Conference. University of Nebraska, Lincoln, Nebraska, USA.

Bowles, M., J. McBride, and T. Bell. 2001. Restoration of the federally threatened Mead's Milkweed (Asclepias meadii). Ecological Restoration 19:235-241.

Bowles, M. L., J. L. McBride, and R. F. Betz. 1998. Management and restoration ecology of the federal threatened Mead's milkweed, Asclepias meadii (Asclepiadaceae). Annals of the Missouri Botanical Garden 85:110-125.

Cormack, R. M. 1964. Estimates of survival from the sighting of marked animals. Biometrika 51:429-438.

Jolly, G. M. 1965. Explicit estimates from capture-recapture data with both death and immigration - stochastic model. Biometrika 52:225-247.

Kettle, W. D., H. M. Alexander, and G. L. Pittman. 2000. An 11-year ecological study of a rare prairie perennial (Asclepias meadii): implications for monitoring and management. The American Midland Naturalist 144:66-77.

Lesica, P., and B. M. Steele. 1994. Prolonged dormancy in vascular plants and implications for monitoring studies. Natural Areas Journal 14:209-212.

Nichols, J. D. 1992. Capture-recapture models. Using marked animals to study population dynamics. BioScience 42:94-102.

Otis, D. L., K. P. Burnham, G. C. White, and D. R. Anderson. 1978. Statistical inference from capture data on closed animal populations. Wildlife Monographs 62:1-135.

Pollock, K. H., J. D. Nichols, C. Brownie, and J. E. Hines. 1990. Statistical inference for capture-recapture experiments. Wildlife Monographs 107:1-97.

Seber, G. A. F. 1965. A note on the multiple recapture census. Biometrika 52:249-259.

Shefferson, R. P., B. K. Sandercock, J. Proper, and S. R. Beissinger. 2001. Estimating dormancy and survival of a rare herbaceous perennial using mark-recapture models. Ecology 82:145-156.

Slade, N. A., and S. M. Blair. 2000. An empirical test of counts of individuals captured as indices of population size. Journal of Mammalogy 81:1035-1045.

Tecic, D. L., J. L. McBride, M. L. Bowles, and D. L. Nickrent. 1998. Genetic variability in the federal threatened Mead's milkweed, Asclepias meadii Torrey (Asclepiadaceae), as 
determined by allozyme electrophoresis. Annals of the Missouri Botanical Garden 85:97-109.

White, G. C. 1999. Program MARK. Version 1.4. Colorado State University, Fort Collins, Colorado, USA.
White, G. C., D. R. Anderson, K. P. Burnham, and D. L. Otis. 1982. Capture-recapture and removal methods for sampling closed populations. Los Alamos National Laboratory, Los Alamos, New Mexico, USA.

\section{APPENDIX}

Each plant generated a series of ones and zeros indicating whether or not the plant was seen in each of eight yearly surveys. For example, a plant seen only in 1993, 1996, and 1998 would be represented by the capture history 01001010 . Using the model described in the methods section and assuming all plants were present in 1992, the year of the first survey considered herein, the probability of this particular capture history would be the product of probabilities of being missed in 1992 (a burn year), surviving to 1993, being seen initially in 1993, surviving to 1994 , being missed but surviving in 1994 and 1995, being resighted in 1996, etc. Not being seen in 1999 could be due to death or surviving and being missed. In many capture models, these two probabilities cannot be estimated separately, but because we assume that the probability of recapture is equal for all years without a burn, the probability of surviving can be estimated for the final year. Formally, the probability of capture history 01001010 was given by

$$
\begin{aligned}
P_{01001010}= & \left(1-C_{b}\right) S_{1} C_{n} S_{2}\left(1-R_{b}\right) S_{3}\left(1-R_{n}\right) \\
& \times S_{4} R_{b} S_{5}\left(1-R_{n}\right) S_{6} R_{b}\left(1-S_{7} R_{n}\right)
\end{aligned}
$$

where $P_{i}$ is the probability of the capture history $i, C_{b}$ is the probability of initial discovery in a year with spring burning, $C_{n}$ is the probability of initial discovery in a year without fire, $R_{b}$ and $R_{n}$ are probabilities of resighting in years with and without fire, and $S_{i}$ is the probability of survival in the year following the $i$ th spring survey. Maximum-likelihood estimators for population size and probabilities of discovery, resighting, and survival were found by maximizing the multinomial likelihood, $L$, given by

$$
L=\left(N ! / \prod x_{i} !\right) \prod P_{i}^{x_{i}}
$$

where $N$ is the population size in $1992, P_{i}$ is the probability of the capture history $i$, and $x_{i}$ is the number of plants with capture history $i$. Our computer program found maximumlikelihood estimates (MLE) by iterating over an 11- or 12dimensional grid of possible values for all parameters. All probabilities started at 0.01 and were incremented by 0.49 , so initially values of $0.01,0.50$, and 0.99 were tried for each. For each combination of values, we calculated likelihoods for each population size equal to the number of plants observed and increasing by integer values up to 1000 plants more than observed. The increment for probabilities was then halved and a new grid searched with initial values set two increments below the value associated with the maximum likelihood. If one of the MLE values was on the edge of the grid, the grid was centered on that value and the search was repeated. Increments were halved until they were smaller than 0.001 . 